Series A

I. MATHEMATICA

542

\title{
ON PAIR ALGEBRAS AND STATE-INFORMATION IN AUTOMATA
}

BY

MAGNUS STEINBY

HEL S INK I 1973

S U O M A L I N EN TIEDEAKATEMIA

https://doi.org/10.5186/aasfm.1973.542 
Copyright (C) 1973 by

Societas Scientiarum Fennica

ISBN 951-41-0110-3

Communicated 8 January 1973 by Arto SalomaA 


\section{Introduction}

In structure theory of sequential machines state-information is usually represented by a partition or cover on the state-set. When discussion is limited to realizations by one-to-one assignments, the partition model appears quite satisfactory both intuitively and mathematically. In their studies summarized in [2], Hartinanis and Stearns employ successfully the lattice of partitions with the substitution property and the notion of partition pairs to describe decompositions and loop-free realizations as well as information-flow in networks of machines.

In order to be able to deal with more general forms of realizations, partitions were replaced by covers (called set systems in [2]), where blocks are allowed to overlap. While many of the central results still hold and some very general results concerning decomposability have been proved (cf. for ex. ZeIGER [4]) using this formalism, the interpretation of covers as stateinformation pose some serious difficulties. Lru [3] has suggested that coverinformation could equivalently be represented by a symmetric, reflexive binary relation. However, it is evident that this simplification of the formalism must involve some loss of detail because the correspondence is not one-to-one. This problem gave the original impetus to this paper. However, it became evident that our questions can be stated and answered in a much more general form. Therefore we first study general lattice morphisms and their effects on pair algebras. The relationship between the two information-representations is then easily described applying the general results.

In the use of terminology we mostly follow BIRkhoff [1]. The discussion has been limited to finite lattices, and the applications therefore to finite automata. Also, some of the results have not been stated in their most general form. Thus, it would sometimes suffice to assume a join or meet morphism instead of a lattice morphism. On the other hand, the results apply with trivial modifications to input-state and the other pair algebras of an automaton not discussed here.

\section{Lattice morphisms}

In the following $K$ and $L$, possibly with subscripts, will always denote finite lattices. The meet and join of two lattice elements $x$ and $y$ is 
written as $x y$ and $x+y$, respectively. Also, inf $\{x: P(x)\}$ and sup $\{x \mid P(x)\}$ denote the infimum and supremum of the elements $x$ having property $P$. The least element is 0 and the greatest element 1 . These notations are used for any lattice, because the lattice will always be known from the context.

Let $h: K \rightarrow L$ be a lattice epimorphism. Then $h^{-1}(u)$ is a sublattice of $K$, for every $u \in L$. The greatest element of this sub-lattice is denoted by $1_{h}(u)$ and the least element by $0_{h}(u)$. When $h$ is understood, we omit the subscripts. The equivalence relation on $K$ induced by $h$ is denoted by $E_{h}$. We may view $0_{h}$ and $1_{h}$ as mappings for which the following lemma is easily verified.

Lemma 2.1. For any lattice epimorphism $h: K \rightarrow L$,

$$
\begin{aligned}
& 0_{h}: L \rightarrow K \text { is a join monomorphism, and } \\
& 1_{h}: L \rightarrow K \text { is a meet monomorphism. }
\end{aligned}
$$

A lattice $K$ is called pseudo-complemented if, for every element $x \in K$, the set of elements $x_{1} \in K$ such that $x x_{1}=0$ contains a greatest element. This element

$$
x^{*}:=\sup \left\{x_{1} \in K: x x_{1}=0\right\}
$$

is then called the pseudo-complement of $x$. In our applications $x^{*}$ will represent the smallest amount of information which combined with $x$ gives perfect state information.

Proposition 2.2. Let $K$ be a pseudo-complemented lattice and $h: K \rightarrow L$ a lattice epimorphism such that $h^{-1}(0)=\{0\}$. Then $L$ is pseudo-complemented and, for any $x, y \in K$,

$$
\begin{gathered}
h(x)^{*}=h\left(x^{*}\right), \\
x E_{h} y \text { implies } x^{*} E_{h} y^{*}, \text { and } \\
x^{*}=1\left(h(x)^{*}\right) .
\end{gathered}
$$

Proof: Let $u \in L$ and $x \in K$ such that $h(x)=u$. Then $u h\left(x^{*}\right)=$ $h\left(x x^{*}\right)=0$. Suppose $u v=0$, for some $v=h(y) \in L$. Then $0=h(x) h(y)$ $=h(x y)$ implies $x y=0$. Therefore, $y \leqq x^{*}, v \leq h\left(x^{*}\right)$ and $h\left(x^{*}\right)=$ $h(x)^{*}$. Statement (2) follows immediately from (1). By the assumption $h^{-1}(0)=\{0\}, h\left(x \mathbf{l}\left(h(x)^{*}\right)\right)=h(x)^{*} \quad h(x)=0$ implies $\mathrm{l}\left(h(x)^{*}\right) \leqq x^{*}$. On the other hand, $h\left(x^{*}\right)=h(x)^{*}$ implies $x^{*} \leqq 1\left(h\left(x^{*}\right)\right)=1\left(h(x)^{*}\right)$. Thus we have verified (3). 


\section{Pair algebras}

A subset $\Gamma \subseteq K_{1} \times K_{2}$, where $K_{1}$ and $K_{2}$ are finite lattic ss is said to form a pair algebra [2] if

(A) for any $x_{1}, x_{2} \in K_{1}$ and $y_{1}, y_{2} \in K_{2},\left(x_{1}, y_{1}\right),\left(x_{2}, y_{2}\right) \in \Gamma$ implies $\left(x_{1}+x_{2}, y_{1}+y_{2}\right),\left(x_{1} \cdot x_{2}, y_{1} \cdot y_{2}\right) \in \Gamma$, and

(B) $(0, y) \in \Gamma$ and $(x, 1) \in \Gamma$, for every $x \in K_{1}$ and $y \in K_{2}$.

We relate pair algebras to the more familiar concept of a subdirect product. A subset $I \subseteq K_{1} \times K_{2}$ is a subdirect product of $K_{1}$ and $K_{2}$ if it satisfies conditon (A) and

(C) $\operatorname{pr}_{1}(\Gamma)=K_{1}$ and $\operatorname{pr}_{2}(T)=K_{2}$, where $\operatorname{pr}_{1}$ and $\operatorname{pr}_{2}$ are the projections to $K_{1}$ and $K_{2}$.

Proposition 3.1: A subset $I \subseteq K_{1} \times K_{2}$ is a pair algebra iff it is a subdirect product and $(0,1) \in \Gamma$.

Proof: Every pair algebra is a subdirect product because condition (B) implies condition (C). Suppose $T$ is a subdirect product and $(0,1) \in I$. For any $x \in K_{1}$, there exists by (C) a pair $(x, y) \in \Gamma$. Using (A) we have $(x, 1)=(x+0, y+1) \in \Gamma$. Dually, $(0, y) \in \Gamma$, for any $y \in K_{2}$.

It is often convenient to consider a pair algebra as a lattice with the naturally defined componentwise operations and partial ordering.

Lemma 3.2. For any lattice epimorphisms $h_{1}: K_{1} \rightarrow L_{1}$ and $h_{2}$ : $K_{2} \rightarrow L_{2}$,

(1) $h:=h_{1} \times h_{2}$ is a lattice epimorphism,

(2) $h(\Gamma)$ is a pair algebra in $L_{1} \times L_{2}$, for any pair algebra $\Gamma \subseteq K_{1} \times K_{2}$, and

(3) $h^{-1}(A)$ is a pair algebra in $K_{1} \times K_{2}$, for any pair algebra $\Delta \subseteq L_{1} \times L_{2}$.

The lemma follows from well known properties of direct products and morphisms. We observe that $h(0,1)=(0,1)$.

For any pair algebra $I \subseteq K_{1} \times K_{2}$, two mappings $\mathrm{m}_{\Gamma}: K_{1} \rightarrow K_{2}$ andi $M_{T}: K_{2} \rightarrow K_{1}$ can be defined as follows. For any $x \in K_{1}$ and $y \in K_{2}$, $m_{\Gamma}(x):==\inf \left\{y_{1} \in K_{2} \mid\left(x, y_{1}\right) \in \Gamma\right\}$, and $M_{I}(y):=\sup \left\{x_{1} \in K_{1} \mid\left(x_{1}, y\right)\right.$ $\in \Gamma\}$.

A number of properties of these mappings can be found in [2]. In the applications where lattice elements represent state information in an automaton, $m_{I} \cdot(x)$ is the maximum next-state information that can be 
computed from the present-state information $x$. Similarly, $M_{\Gamma}(y)$ is the minimum present-state information required for the next-state information $y$.

Proposition 3.3. Let $h_{1}: K_{1} \rightarrow L_{1}, h_{2}: K_{2} \rightarrow L_{2}$ be lattice epimorphisms and $\Gamma \subseteq K_{1} \times K_{2}, \Delta \subseteq L_{1} \times L_{2}$ pair algebras such that $h_{1} \times h_{2}(\Gamma)=\Delta$. Then

$$
\begin{aligned}
m_{\Delta}(u) & =h_{2}\left(m_{\Gamma}(0(u))\right), \text { for any } u \in L_{1}, \\
M_{\Delta}(v) & =h_{1}\left(M_{\Gamma}(\mathrm{l}(v))\right), \text { for any } v \in L_{2}, \\
h_{2}\left(\left(m_{\Gamma}(x)\right)\right. & \geqq m_{\Delta}\left(h_{1}(x)\right), \text { for any } x \in K_{1}, \text { and } \\
h_{1}\left(M_{l}(y)\right) & \leqq M_{\Delta}\left(h_{2}(y)\right), \text { for any } y \in K_{2} .
\end{aligned}
$$

Proof: Because the first two statements are dual to each other (in a generalized sense), we prove (1) only. We claim that $(u, v) \in \mathcal{A}$ iff there exists a $y \in K_{2}$ such that $h_{2}(y)=v$ and $(0(u), y) \in I$. Suppose $y$ satisfies these conditions. Then

$$
(u, v)=\left(h_{1}(0(u)), h_{2}(y)\right) \in h_{1} \times h_{2}(\Gamma)=1 .
$$

Conversely, let $(u, v) \in \Delta$. Then there exists a pair $(x, y) \in \Gamma$ such that $h_{1} \times h_{2}(x, y)=(u, v)$. But $h_{1}(x)=u$ implies $\theta(u) \leq x$ and $(0(u), y) \in \Gamma$. Also $h_{2}(y)=v$. Using this result we have

$$
\begin{aligned}
h_{2}\left(m_{I}(0(u))\right) & =\inf \left\{h_{2}(y) \mid(0(u), y) \in I\right\} \\
& =\inf \{v \mid(u, v) \in \mathcal{A}\} \\
& =m_{\Delta}(u) .
\end{aligned}
$$

Statement (3) follows from (1) because $x \geq 0\left(h_{1}(x)\right)$ and both $h_{2}$ and $m_{I}$, are isotone mappings. Similarly, (4) follows from $(2)$.

Corollary 3.3.1. With the assumptions of Proposition 3.3, we have

$$
\begin{aligned}
& 0\left(m_{\Delta}(u)\right) \leqq m_{\Gamma}(0(u)), \text { for any } u \in L_{1} \text {. and } \\
& \mathbf{l}\left(M_{\Delta}(v)\right) \geqq M_{\Gamma}(1(v)), \text { for any } v \in L_{2} .
\end{aligned}
$$

We shall now show that these results can be strengthener when we make some additional assumptions concerning $h_{1}$ and $h_{2}$. In particular, we can compute $m_{\Delta}$ and $M_{\Delta}$ in the pair algebra $I^{\prime}$ using any element from the corresponding $E_{h_{1}}$ - or $E_{h_{2}}$ - class.

Proposition 3.4. Let $h_{1}: K_{1} \rightarrow L_{1}, \quad h_{2}: K_{2} \rightarrow L_{2}$ be lattice epimorphisms and $\Gamma \subseteq K_{1} \times K_{2}, \Delta \subseteq L_{1} \times L_{2}$ pair algebras such that $h_{1} \times h_{2}(\Gamma)=A$ and, for any $x \in K_{1}, v \in L_{2}$, 
(a)

$$
(x, 1(v)) \in \Gamma \text { if }\left(h_{1}(x), v\right) \in A .
$$

Then

$$
\begin{gathered}
m_{\Delta}\left(h_{1}(x)\right)=h_{2}\left(m_{I^{\prime}}(x)\right), \text { for any } x \in K_{1}, \\
1\left(M_{\Delta}(v)\right)=M_{\Gamma^{\prime}}(1(v)), \text { for any } v \in L_{2}, \\
1\left(m_{\Delta}(u)\right) \geqq m_{\Gamma^{\prime}}(1(u)), \text { for any } u \in L_{1} \text {, and } \\
x_{1} E_{h_{1}} x_{2} \text { implies } m_{\Gamma^{\prime}}\left(x_{1}\right) E_{h_{2}} m_{\Gamma^{\prime}}\left(x_{2}\right) \text {, for any } x_{1}, x_{2} \in K_{1} .
\end{gathered}
$$

Proof: Statement (1) can be proved using (a) as follows. If $(x, y) \in \Gamma$, then $\left(h_{1}(x), h_{2}(y)\right) \in \Delta$. Conversely, let $\left(h_{1}(x), v\right) \in \Delta$. Then $v=h_{2}(y)$ and $(x, y) \in \Gamma$ if we choose $y=1(v)$. Hence,

$$
\begin{aligned}
h_{2}\left(m_{\Gamma}(x)\right) & =\inf \left\{h_{2}(y) \mid(x, y) \in \Gamma\right\} \\
& =\inf \left\{v \mid\left(h_{1}(x), v\right) \in A\right\} \\
& =m_{\Delta}\left(h_{1}(x)\right) .
\end{aligned}
$$

Consider now any $v \in L_{2}$ and let $x=1\left(M_{\Delta}(v)\right)$. Then $\left(M_{\Delta}(v), v\right) \in \Delta$ implies $\left(1\left(M_{\Delta}(v)\right), 1(v)\right) \in \Gamma$ by condition (a). Therefore, $1\left(M_{\Delta}(v)\right) \leq$ $M_{I^{\prime}}(1(v))$. Combining this with Corollary 3.3.1 we get $(2)$. Let $v \in L_{1}$. Writing $x=1(u)$ in (1), we get $m_{\lrcorner}(u)=h_{2}\left(m_{\Gamma}(1(u))\right)$. Therefore, $m_{\Gamma}(1(u))$ $\leq 1\left(m_{\Delta}(u)\right)$. Statement (4) follows easily from (1).

We note that statement (4) in Proposition 3.4 means that the $m_{\Gamma^{\prime}}$ operator can be computed in the image algebra "modulo $E_{h_{2}}$ " assuming that the argument is known "modulo $E_{h_{1}}$ ". Assumption (a) is equivalent to the condition that $(1(u), 1(v)) \in \Gamma$ whenever $(u, v) \in \Delta$.

The following results can be obtained similarly as Proposition 3.4.

Proposition 3.5. Let $h_{1}: K_{1} \rightarrow L_{1}, h_{2}: K_{2} \rightarrow L_{2}$ be lattice epimorphisms and $I \subseteq K_{1} \times K_{2}, \Delta \subseteq L_{1} \times L_{2}$ pair algebras such that $h_{1} \times h_{2}(\Gamma)=\Delta$ and, for any $u \in L_{1}$ and $y \in K_{2}$,

$$
(0(u), y) \in \Gamma \text { if }\left(u, h_{2}(y)\right) \in \mathcal{A}
$$

Then

(4) $y_{1} E_{h_{2}} y_{2}$ implies $M_{\Gamma}\left(y_{1}\right) E_{h_{1}} M_{\Gamma^{r}}\left(y_{2}\right)$, for any $y_{1}, y_{2} \in K_{2}$. 
Again, we note that under condition (b) $M_{\Gamma}$ can be computed in $\Delta$ "modulo $\mathrm{E}_{h_{1}}$ ". The condition (b) means that $(0(u), 0(v)) \in \Gamma$ whenever $(u, v) \in \Delta$.

We conclude this section by the following result which can easily be proved.

Proposition 3.6. With the notations and combined assumptions of Propositions 3.4. and 3.5., $T \cap\left(h_{1}^{-1}(u) \times h_{2}^{-1}(v)\right)$ is a pair algebra in $h_{1}^{-1}(u) \times$ $h_{2}^{-1}(v)$, for any $u \in L_{1}, v \in L_{2}$.

\section{Covers and relations}

Let $Q$ be a finite set. A collection $\alpha$ of subsets of $Q$ is called a cover (or set system in [2]) if $\mathrm{U}\{A \mid A \in \alpha\}=Q$ and $A_{1} \supseteq A_{2}$ implies $A_{1}=A_{2}$, for any $A_{1}, A_{2} \in \alpha$. The set of all covers of $Q$ is denoted by $C_{Q}$. For any $\alpha, \beta \in C_{Q}$, we write

$$
\alpha \leq \beta \text { iff }(\forall A \in x)(\text { G } B \in \beta) A \subseteq B .
$$

Then $C_{Q}$ becomes a distributive lattice, ef. [2], where

$$
\alpha \cdot \beta=\max \{A \cap B \mid A \in \alpha, B \in \beta\}
$$

and

$$
\alpha+\beta=\max \{\alpha \cup \beta\}
$$

The least cover 0 consists of singletons only, and the greatest cover is $1=\{Q\}$.

Let $R_{Q}$ denote the set of all symmetric, reflexive relations on $Q$. If we order the relations by the usual inclusion relation, $R_{Q}$ becomes a Boolean lattice, where $\varrho \cup \sigma$ and $\varrho \cap \sigma$ are the join and meet of $\varrho, \sigma \in R_{Q}$.

The greatest element is $Q \times Q$ and the least element the equality relation $\varepsilon$.

Following LIU [3] we assign to every $x$ in $C_{Q}$ the relation $r(x):=$ $\left\{\left(q, q^{\prime}\right) \mid\right.$ ( $\left.\left.A \in A \in\right) q, q^{\prime} \in A\right\}$.

Clearly, we get a mapping from $C_{Q}$ to $R_{Q}$, but it is not one-to-one, if $Q$ has more than two elements.

Proposition 4.1. For any set $Q, r: C_{Q} \rightarrow R_{Q}$ is a lattice epimorphism. For any $\varrho \in R_{Q}$, the sublattice $r^{-1}(\varrho)$ has the least element

$$
0_{r}(\varrho)=\max \left\{\left\{q, q^{\prime}\right\} \mid\left(q, q^{\prime}\right) \in \varrho\right\},
$$


and the greatest element

$$
1_{r}(\varrho)=\max \{C \mid C \times C \subseteq \varrho\}
$$

In particular, $r^{-1}(\varepsilon)=\{0\}$.

We om it the straightforward proof.

Proposition 4.2. For any set $Q$,

$$
\begin{gathered}
0_{r}: R_{Q} \rightarrow C_{Q} \text { is a lattice monomorphism, and } \\
1_{r}(\varrho \cap \sigma)=1_{r}(\varrho) \cdot 1_{r}(\sigma), \text { for any } \varrho, \sigma \in R_{Q} .
\end{gathered}
$$

This proposition is a special case of Lemma 2.1. except for the claim that $0_{r}$ is a meet morphism. This again follows from the following observation. Let $C_{Q 2}$ be the set of all covers of $Q$ with no blocks containing more than two elements. Clearly, $C_{Q 2}$ is a sublattice of $C_{Q}$ and $r$ restricted to $C_{Q^{2}}$ is an isomorphism with $0_{r}$ as its inverse.

Because $C_{Q}$ and $R_{Q}$ are finite distributive lattices, they certainly are pseudo-complemented. Using Proposition 2.2. and the last part of Proposition 4.1., we get the following result.

Proposition 4.3. For any $\alpha, \beta \in C_{Q}$,

$$
\begin{aligned}
\alpha \beta & =0 \text { iff } r(\alpha) \cap r(\beta)=\varepsilon, \\
r\left(\alpha^{*},\right. & =r(\alpha)^{*}, \text { and } \\
\alpha^{*} & =1\left(r(\alpha)^{*}\right) .
\end{aligned}
$$

In order to interprete these results, we assume that $Q$ is the state set of an automaton $(Q, X, T)$, where $X$ is the set of input symbols and $T: Q \times X \rightarrow Q$ the transition function. We will call this automaton simply $T$. For any $q \in Q$ and $x \in X$, we use the notation $q x^{T}:=T(q, x)$.

Every cover $\alpha \in C_{Q}$ represents now some information about the state of $T$. Similarly, each relation $\varrho \in R_{Q}$ can be viewed as some state-information (cf. [3]). It follows from Proposition 4.1. that the relation representation of some state-information determines its cover representation with the accuracy of a sublattice of $C_{Q}$ only. On the other hand, this correspondence between the two representations is preserved in the combination of information (meet) and the combination of ignorance (join). The pseudo-complement $\alpha^{*}$ represents the minimum information which combined with $\alpha$ gives perfect state information. Proposition 4.3 tells us that this can essentially be computed in $R_{Q}$ using $r(\alpha)$. Also, the question whether the cover information $\alpha$ and $\beta$ jointly give perfect state information can be resolved considering the relational equivalents. 
(We have ignored here the difficulties involved in the interpretation of $x \beta$ as the combined information, cf. [2]). In general, the combined information $\alpha \beta$ can be computed in $R_{Q}$ "modulo $E_{r}$ " only.

\section{Relation pairs and cover pairs}

From here on $Q$ will always be the state set of the automaton $T$. The pair $(\alpha, \beta) \in C_{Q} \times C_{Q}$ is called a cover pair (system pair in [2]) if, for every $A \in \alpha$ and $x \in X$ there exists a block $B \in \beta$ such that $A x^{T}:=$ $\left\{q:\left(\mathbb{A} q^{\prime} \in A\right) q^{\prime} x^{T}=q\right\} \subseteq B$. The set of cover pairs $C P_{T}$ of $T$ forms a pair algebra, ef. [2].

We call $(\varrho, \sigma) \in R_{Q} \times R_{Q}$ a relation pair if, for any $q, q^{\prime} \in Q$ and $x \in X,\left(q, q^{\prime}\right) \in \varrho$ implies $\left(q x^{T}, q^{\prime} x^{T}\right) \in \sigma$. The set of all relation pairs of $T$ is denoted by $R P_{T}$.

Proposition 5.1. For any automaton $T, R P_{T}$ is a pair algebra in $R_{Q} \times R_{Q}$.

We omit the simple proof. The $m$ - and $M$-operators can now be introduced as usual. They are denoted by $m_{C}$ and $M_{C}$ in $C P_{T}$, and by $m_{R}$ and $M_{R}$ in $R P_{T}$. For any $\varrho \in R_{Q}$, we can evidently compute $m_{R}(\varrho)$ and $M_{R}(\varrho)$ as follows:

$$
m_{R}(\varrho)=\left\{\left(q x^{T}, q^{\prime} x^{T}\right) \mid\left(q, q^{\prime}\right) \in \varrho, x \in X\right\} \cup \varepsilon
$$

and

$$
M_{R}(\varrho)=\left\{\left(q, q^{\prime}\right) !\left(q x^{T}, q^{\prime} x^{T}\right) \in \varrho, x \in X\right\}
$$

Proposition 5.2. For any automaton $T$ and $\varrho, \sigma \in R_{Q}$,

$$
\begin{gathered}
r \times r\left(C P_{T}\right)=R P_{T}, \\
(\varrho, \sigma) \in R P_{T} \text { iff }(0(\varrho), 0(\sigma)) \in C P_{T} \text {, and } \\
(\varrho, \sigma) \in R P_{T} \text { iff }(1(\varrho), 1(\sigma)) \in C P_{T} .
\end{gathered}
$$

Proof: We first prove that $(\alpha, \beta) \in C P_{T}$ implies $(r(\alpha), r(\beta)) \in R P_{T}$. This gives one half of all of the statements. Thus, let $\left(q, q^{\prime}\right) \in r(\alpha)$ and $x \in X$. Then $q, q^{\prime} \in A$, for some $A \in x$ such that $A x^{T} \subseteq B$ where $B \in \beta$. This implies $\left(q x^{T}, q^{\prime} x^{T}\right) \in r(\beta)$ as required. To prove the second part of $(2)$, we have to show that $(\varrho, \sigma) \in R P_{T}$ implies $(0(\varrho), 0(\sigma)) \in C P_{T}$. Now every block of $0(\varrho)$ is of the form $\left\{q, q^{\prime}\right\}$, where $\left(q, q^{\prime}\right) \in \varrho\left(q=q^{\prime}\right.$ possible). For any $x \in X,\left(q x^{T}, q^{\prime} x^{T}\right) \in \sigma$, and hence $\left\{q x^{T}, q^{\prime} x^{T}\right\} \subseteq B$, for some $B \in 0(\sigma)$. Statement (2) implies $R P_{T} \subseteq r \times r\left(C P_{T}\right)$, and thus 
we have the second half of (1). Finally, let $(\varrho, \sigma) \in R P_{T}$, and $A \in 1(\varrho)$, $x \in X$. Then $A \times A \subseteq \varrho$, and $\left(A x^{T}\right) \times\left(A x^{T}\right) \subseteq \sigma$. Hence, $A x^{T} \subseteq B$ for some $B \in 1(\varrho)$. This proves statement (3).

It follows from Proposition 5.2. that all results of Section 3 are applicable here.

A relation $\varrho \in R_{Q}$ is called an SP relation (SP for substitution property) if $(\varrho, \varrho) \in R P_{T}$. Similarly, SP covers are defined.

Corollary 5.2.1. For any automaton $T$ and $\varrho \in R_{Q}$, the following three conditions are equivalent:

$$
\begin{gathered}
\varrho \text { is an SP relation, } \\
0(\varrho) \text { is an SP cover, and } \\
\mathrm{l}(\varrho) \text { is an SP cover. }
\end{gathered}
$$

This result cannot be extended to apply to any $x \in h^{-1}(\varrho)$.

The next corollary follows from statements (1) and (3) of Proposition 3.4 , and statement (2) of Proposition 3.5.

Corollary 5.2.2. For any automaton $T, \alpha, \beta \in C_{Q}$ and $\varrho \in R_{Q}$,

$$
\begin{aligned}
& m_{R}(r(\alpha))=r\left(m_{C}(\alpha)\right), \\
& 1\left(m_{R}(\varrho)\right) \geqq m_{C}(1(\varrho)), \\
& 0\left(m_{R}(\varrho)\right)=m_{C}(0(\varrho)), \text { and } \\
& \chi E_{r} \beta \text { implies } m_{C}(\chi) E_{r} m_{C}(\beta) .
\end{aligned}
$$

Similarly, we get the following results.

Corollary 5.2.3. For any automaton $T, \lambda, \beta \in C_{Q}$ and $\varrho \in R_{Q}$,

$$
\begin{aligned}
& M_{R}(r(\alpha))=r\left(M_{C}(\curlywedge)\right), \\
& 0\left(M_{R}(\varrho)\right) \leqq M_{C}(0(\varrho)), \\
& 1\left(M_{R}(\varrho)\right)=M_{C}(1(\varrho)), \text { and } \\
& \alpha E_{r} \beta \text { implies } M_{C}(\imath) E_{r} M_{C}(\beta) .
\end{aligned}
$$

\section{Concluding remarks}

As a conclusion we comment on some aspects of the previous results which may be of significance to the structure theory of sequential machines. 
We have seen that the algebra of symmetric, reflexive relations is an epimorphic image of the algebra of covers. Thus relation information provides a representation of cover information, although not a faithful one. Using Corollaries 5.2.2 and 5.2.3 we can compute $m_{R}(\varrho)$ and $M_{R}(\varrho)$ in the cover algebra using any $\alpha \in r^{-1}(\varrho)$ as the argument:

$$
m_{R}(\varrho)=r\left(m_{C}(\alpha)\right)
$$

and

$$
M_{R}(\varrho)=r\left(M_{C}(\alpha)\right) \text {. }
$$

On the other hand, $m_{C}$ and $M_{C}$ can be computed "modulo $E_{r}$ using the relation representation even if the cover is known »modulo $E_{r}$ only. Thus the possible loss of information resulting when we transfer from the cover representation to the relation representation will not spread during computations.

From Corollary 5.2.1 we see that, for any serial decomposition which could be found using covers, there exists a serial decomposition which can be detected from the lattice of SP relations. Using Proposition 4.3 too, we get the same result for parallel decompositions.

These observations suggest that the cover representation could sometimes be replaced by a narrower formalism based on symmetric, reflexive relations without any essential loss power.

\section{Acknowledgement}

This work has been supported by Grant A7911 of the National Research Council of Canada.

Department of Computer Science

University of Western Ontario

London, Canada 


\section{References}

[1] Birkhoff, G.: Lattice Theory, Amer. Math. Soc., Providence, Rhode Island (1967).

[2] Hartmanis, J. and Stearns, R. E.: Algebraic Structure Theory of Sequential Machines, Prentice-Hall, Englewood Cliffs, New Jersey (1966).

[3] Lrv, C. L.: Lattice Functions, Pair Algebras and Finite-state Machines, J. Assoc. Comput. Mach. 16 (1969), $442-454$.

[4] Zeiger, H. P.: Cascade synthesis of finite-state machines, Information and Control 10 (1967), 419-433. 